\title{
External and body-centered frames of reference in spatial memory: Evidence from touch
}

\author{
SUSANNA MILLAR and ZAINAB AL-ATTAR \\ University of Oxford, Oxford, England
}

\begin{abstract}
The study reports independent effects of external and body-centered reference cues on spatial coding of an irregular sequence of haptic locations. The aim was to investigate the nature of spatial coding by using a modality that does not provide distal cues routinely. Our method isolates and combines body-centered and external spatial reference cues for irregularly placed locations, scanned along a raised-line route. Disrupting body-centered reference for the locations, by orienting the map differently to the body in the test phase than in the presentation phase, doubled errors in positioning the locations along the route in recall. Adding external reference, by giving instructions to use a surrounding frame for reference when body-centered coding was disrupted, reduced errors to near baseline (no-rotation) levels. Adding external reference cues to intact (not displaced) body-centered reference halved errors, as compared with the baseline. The results are consistent with the hypothesis that accurate spatial coding is determined by the congruence of potential reference cues from diverse sources. The new findings suggest that external and body-centered reference cues have independent additive effects on spatial coding. The sequence of locations had a significant effect in all the reference conditions, suggesting the additional use of fortuitous but distinctive local touch cues on the route. The discussion considers theoretical and practical implications of the results.
\end{abstract}

This study concerns the processes by which spatial coding takes place. The nature of spatial processes and how they relate to each other is a central question for theoretical descriptions of spatial coding in immediate memory. In most studies in this area, spatial tasks in vision have been used. A series of studies by the first author adopted the alternative approach of using spatial tasks with touch and movement, in the total absence of vision (Millar, 1975, 1976, 1979, 1981, 1985, 1991, 2000). These studies suggested that the important factor in spatial coding is the availability of reference information. Spatial coding is characterized by processing inputs as reference cues that can specify the location, distance, or direction responses that a task requires. The reference hypothesis assumes further that accurate spatial coding is determined by the congruence of inputs from external and from body-centered sources that provide potential reference cues (Millar, 1988, 1994). There is little direct evidence so far on the relation of external reference and body-centered reference cues in spatial coding and none, as far as we know, for inputs from touch and movement without vision. Our method separates and combines body-centered and external reference cues to test spatial coding of an irregularly placed sequence of loca-

Correspondence concerning this article should be addressed to S. Millar, Department of Experimental Psychology, University of Oxford, South Parks Road, Oxford OX1 3UD, England (e-mail: susanna. millar@psy.ox.ac.uk).

Note-This article was accepted by the previous editorial team, headed by Neil Macmillan. tions, analogous to spatial displays in visuospatial studies, but scanned without vision along a felt route.

The distinction between body-centered (egocentric) and external (allocentric) reference classifies multiple inputs very broadly by their source (e.g., Barrett, Bradshaw, Rose, Everatt, \& Simpson, 2001; Berthoz, 1991; Paillard, 1991; Pashler, 1990; Stein, 1992). It is easy to locate an object, felt previously without vision, if your body is still in the same position relative to that location. The reference is lost if you move or if someone changes the location of the object relative to your body. External reference involves specifying the location of the target relative to cues beyond, or surrounding, the target. You can locate your pen accurately on the desk if its position is specified relative to desktop cues, even if you have moved or the orientation of the desk has been changed.

Our question was about the reference cues that people use to recall locations, rather than about internal coordinate frames or mental representation. We therefore used a tactile map in tabletop space with raised lines that provided guiding routes for the direction of scanning, so that our task did not require mental rotation of directions. The use of body-centered (gravitational, proprioceptive, midline, or other posture) cues for reference would be shown if the accuracy of recall depended on maintaining the body-to-target relation and would be lost if that relation was disrupted, even if the direction of change did not have to be computed.

We provided external reference information by an actual (raised-line) surrounding frame. We are grateful to an anonymous reviewer for alerting us to a visual study (Hin- 
ton \& Parsons, 1988) that used the tabletop surround as the external (scene-based) reference frame, analogous to the palpable frame used here. In the visual study, external cues from the desktop environment acted as external reference frames and could be distinguished from viewercentered cues. In our study, the participants were instructed to use one hand for the target and the other hand concomitantly for the frame and to relate the target location to frame cues.

Quite young children, as well as adults, are able to understand instructions to use reference cues (e.g., Ungar, Blades, \& Spencer, 1997). We have used surrounding frames before (Millar, 1985; Millar \& Al-Attar, 2000, 2001). However, to our knowledge, neither these studies nor other, previous studies in purely haptic conditions have provided evidence on the relative effects of body-centered and external reference cues.

The question was whether, when body-centered reference was disturbed, instructions to use the external frame for reference in haptic conditions would produce more accurate responses than would the absence of external cues and instructions.

\section{Alternative Hypotheses and Predictions}

An influential traditional view of spatial coding has been that spatial organization differs radically in haptics (active touch) and vision: Haptic space is centered on the body, whereas vision depends on external spatial coordinates (Hatwell, 1960; Revesz, 1950; Warren, 1977). A somewhat similar view is suggested by use of the metaphor of a visuospatial sketchpad to exemplify the immediate registration of spatial information in working memory (Baddeley, 1990; Baddeley \& Hitch, 1974; but see Baddeley, 2000).

There is evidence that body-centered reference is important in spatial task performance with haptic inputs. Symmetry is not a salient feature in the recognition of haptic shapes, but it facilitates shape recognition if the scanning fingers are aligned with the body midline (Ballesteros, Millar, \& Reales, 1998; Millar, 1978). Haptic inputs depend on scanning movements as much as on touch. They are more accurate in conditions that specify distances or directions by reference to cues from body postures (Hasbrouq \& Guiard, 1986; Hatwell, 1960; Heller, Calcaterra, Green, \& Barnette, 1999; Millar, 1975, 1979, 1981, 1985). The length and direction of movements in recall can have significant distorting effects when the target location is not well specified (Adams \& Dijkstra, 1966; Laabs, 1973; Laabs \& Simmons, 1981; Lechelt, 1982; Millar, 1979, 1981, 1985; Millar \& Ittyerah, 1991). Changing the movement made to reach for a target produces errors. But these are significantly lower when the body-to-target relation remains the same than when that is changed also (e.g., Millar, 1981, 1985). The contrast with vision seems obvious. Vision provides external background cues that can specify the location of objects, whether or not bodycentered cues are also used for reference.

Nevertheless, the view that spatial coding of haptic inputs is confined to body-centered (egocentric) reference and that vision (or some distal sense) is necessary for external (allocentric) reference has implications that require direct testing. The difference between testing the types of mental representations and questions about the use of information from the diverse sources will be briefly considered first.

Individual differences in mental spatial representation and spatial ability with age, visual experience, gender, or cortical damage have frequently been investigated with tasks that require mental rotation of directions. The point of the test is to indicate the new direction of a location "as the crow flies" by mentally computing the new direction from a rotated vantage point or display. This can produce typical direction errors (e.g., Rossano \& Warren, 1989b). Formally, spatial tasks can be described by Euclidean geometric principles. In fact, strict application of Euclidean principles seems to be relatively rare, except in special (test or professional) contexts. People tend to use quite reasonable, although fallible, heuristics derived from experience (e.g., Helstrup, 2001; see also Kahneman, Slovic, \& Tversky, 1982). Testing the time course and errors in the recognition of unfamiliar rotated shapes has suggested the use of visual imagery, or mental manipulations analogous to actually rotating shapes, objects, or bodies (Shepard \& Cooper, 1982; Shepard \& Metzler, 1971), rather than the strict application of geometric rules. Vision is not, in fact, necessary to solve spatial problems, although performance is often poorer in blind conditions (e.g., Carpenter \& Eisenberg, 1978; Hollins \& Kelley, 1988; Millar, 1988, 1994, 2000; Rossano \& Warren, 1989a). But that was not the issue here. Spatial coding is not possible unless some actual stimulus information is present and used (Hinton \& Parsons, 1988). In this study, we asked what cues people actually use for reference with purely haptic inputs.

The hypothesis that spatial coding divides along modality-specific lines differs in that respect from the reference hypothesis mentioned in the introduction. The difference is testable. If haptic inputs can be coded spatially only by body-centered reference, adding instructions to use cues from an actual surrounding frame to specify the target location should not increase accuracy beyond the level found with body-centered reference alone. More important, errors due to the disruption of body-centered reference should be the same whether or not external frame information is added. A further possibility is that instructions to use external cues might actually interfere with body-centered coding.

The reference hypothesis assumes, instead, that the form of spatial coding depends on the balance of actual inputs from the diverse sources that can be used for reference in different task conditions. Body-centered (egocentric) reference is used for haptic inputs because external background cues are not normally available in haptic conditions. However, coding in terms of external (allocentric) reference should occur if the information is provided explicitly. The hypothesis, therefore, predicts that coding is significantly more accurate when instructions to use an external 
frame for reference are added to body-centered reference than without that information. Furthermore, errors due to the disruption of body-centered reference should be significantly reduced by providing external frame information.

We used an unpredictably placed sequence of locations to ensure that the locations did not form an easy regular pattern. One reason for using an unpredictably placed sequence was to use spatial layouts similar to those used in studies of visuospatial coding (Baddeley, 1990). But an irregular pattern of locations was also needed to test the effects of the two forms of reference, because so far, we have evidence on body-centered coding in touch only for single locations. It cannot be assumed that effects are necessarily the same for a sequence of locations.

The present study was designed to test the possible relations between the two forms of reference that are predicted by the alternative hypotheses. The predictions require several comparisons between reference conditions (see below).

\section{Experimental Manipulation of Reference Cues}

The method adopted here was to use what is intuitively an obviously spatial task but to test spatial coding independently of the task by varying body-centered and external frame information experimentally. We used a tactile street map that had raised lines for streets and side streets that formed an irregular route. We placed five locations (one less than the average verbal or visual span), represented by palpable landmark symbols, on the raised route that was to be followed, dispersed in a chance fashion so that they did not form an easy regular pattern, as in visuospatial paradigms (see above).

The use of body-centered reference for coding the locations was tested by comparing the accuracy of recall when the map-to-body relation remained the same in recall as in presentation with accuracy in conditions in which that relation was disturbed by aligning the map differently in recall. It is important to stress again that the task did not require mental rotation of directions. Only the location of the target on the route had to be recalled. The direction of the route was always indicated, initially by experimenter-guided scanning along a small path and then by following the felt raised-line route ("as if walking along the street"), which also provided felt guiding lines for the direction of scanning when the map was oriented differently to the body. The difference is important, because testing for body-centered reference involved changing the relative map-to-body orientation in some conditions (see above). But the sole point of displacing the map here was to disrupt body-centered reference, in order to compare it with other reference conditions. The route in the changed map orientation was, therefore, always indicated, as in the other conditions. External reference was provided by instructions to relate the locations to a palpable surrounding frame (see above). The dependent measure in assessing the effect of the different reference conditions was, therefore, the accuracy with which the participants stopped at the remembered location on the route in recall.
Studies with haptic displays sometimes use screens that prevent sight of the hand and target (e.g., Johnson, 1982; Oldfield \& Philips, 1983). However, such screens do not exclude visual cues from the device itself or from the background beyond the screen. In order to be sure that visual cues could neither help nor interfere with performance, the participants were blindfolded.

\section{EXPERIMENTS}

The predictions from the two hypotheses required comparisons between the following four reference conditions: (1) Body-centered cues could be used for reference, but there were no external cues; (2) body-centered reference was disrupted, and there were no external cues; (3) bodycentered reference was disrupted, but external reference information was provided; and (4) body-centered cues, as well as cues from an external frame, could be used for reference.

The four reference conditions were tested in separate experiments, each with 10 new participants. The detailed procedures for the four reference conditions and an overall analysis of variance (ANOVA) of all the data will be reported before the comparisons that tested the two hypotheses.

\section{Method}

\section{Design and Participants}

A reference conditions (4) $\times$ locations (5) design (repeated measures on the last factor) was used. The task was to remember the location of each of five landmarks, irregularly positioned on a complex route, and to stop at these locations on a test map that did not provide landmarks for the locations. Forty right-handed volunteers were recruited from sixth-form (final high school year), undergraduate, and young graduate students for the study. Ten ( 6 female and 4 male) new participants were allocated randomly to each of the four experimental reference conditions. The effects of gender and number of trials (five in each condition) were examined, but no significant effects were found.

\section{Materials}

We used a $(20 \times 20 \mathrm{~cm})$ raised-line map, based on part of an actual street map, for all presentations of the designated route (Figure 1). The map was simplified in order to increase tactual clarity but provided a relatively realistic context for the task. The designated route included a main east-west road, represented by two parallel plain lines, divided by two crossings $(0.5-\mathrm{cm}$ gaps $)$, and angled $\left(45^{\circ}\right.$ and $135^{\circ}$ ) side roads ( 6 and $8 \mathrm{~cm}$, represented by broken lines) and paths ( 3 and $3.5 \mathrm{~cm}$, represented by dotted lines). Six easily discriminable shapes were selected as landmarks. One (bus station) was used as the start (not counted). The other five landmarks were placed at irregular intervals on the route to avoid a spatially regular or predictable pattern (see above). Neither the map, nor the route, nor the locations were not familiar to any of the participants.

Guided scanning in the correct direction was along the small dotted path from the bus station to the start of the broken-line side road. Independent scanning of the five locations on the designated route started at this point. The to-be-remembered locations were reached in the following order: (1) bank (south line of the main road, located $2 \mathrm{~cm}$ from the east); (2) supermarket (connected at $2.5 \mathrm{~cm}$ from the east to the north line of the main road); (3) railway (located at the end of the 6-cm side road); (4) friend's house $(3.8 \mathrm{~cm}$ west of the first 


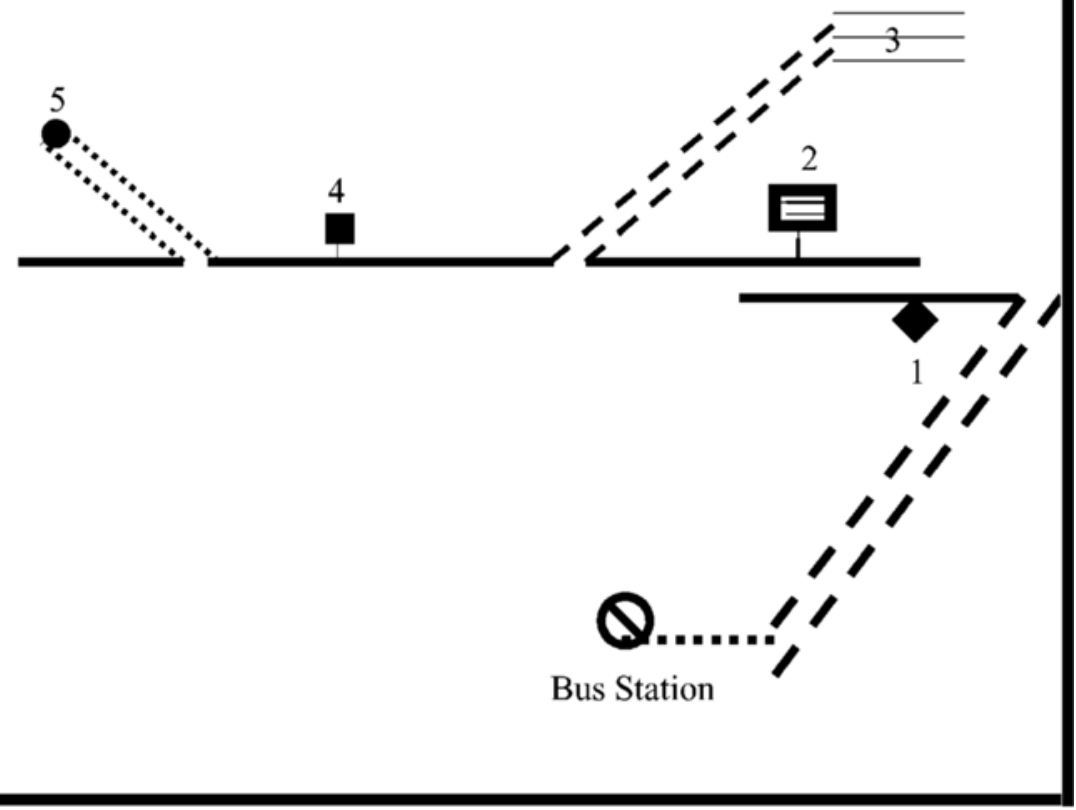

Figure 1. The designated route in presentation, with the symbols for the landmarks for the bus station (start) and at Locations 1 (bank), 2 (supermarket), 3 (railway station), 4 (friend's house), and 5 (home). Neither numbers nor labels appeared on the tactile display. The surrounding square $(20 \times 20 \mathrm{~cm})$ raised-line frame was scanned only in E-frame conditions (see the text).

crossing on the north line of the main road); and (5) home (at the end of the $3.5-\mathrm{cm}$ side path at the second crossing). The $20 \times 20 \mathrm{~cm}$ square raised-line frame, surrounding the map, was used only in external frame (E-frame) conditions.

The test map was identical but did not contain landmark shapes or lines leading to these. Both maps were printed in black on white and were embossed professionally (by the Royal National Institute for the Blind) on swell paper by a process that produces easily felt, clear raised lines and shapes from the black lines and areas on the designs. The map was subsequently marked lightly in pencil with grid (ruler) lines that could not be felt (or seen) by the participant (see the Scoring section).

\section{Procedures}

The participants were blindfolded prior to any demonstration and throughout the trials. They were tested individually, seated at the table on which the display was presented. The task was described as one of using a tactile map. The participants were told to remember the precise location of each landmark on a designated route so that they could later indicate each location on a test map on which the locations were not marked.

In all the presentations, the center of the map on the table was aligned with the participant's body midline. The participants were asked to use their preferred (right) hand index finger for scanning. In all the presentation and test conditions, the experimenter placed the scanning finger at the beginning and indicated the direction in which the route was to be scanned by initially guiding the finger along the small dotted path to the start of the route.

The experimenter demonstrated the route first by describing the symbols used for the roads, paths, and landmarks while the participants scanned these by touch. A second demonstration described the route leading to the sequential landmarks, using a meaningful context (to the bank, then shopping, going to meet a friend in the railway station, and returning home via the friend's house), while the participants scanned the route.

There were five experimental trials in each condition. Each trial consisted of scanning the presentation route with the landmarks at the correct locations, followed by scanning the route on the test map without landmarks. The locations were thus presented five times, providing feedback on the correct locations over trials. The participants were told to place the fingertip on the middle of each landmark symbol so that the midpoint of the ball of the finger touched the middle of the landmark symbol. They were asked to remember the midpoint of that location on the route so that they could place their finger precisely at that point in tests in which the landmark was not present. The need to remember the precise location of each landmark was stressed during all the demonstrations and prior to each presentation.

Recall tests followed immediately (approximately $3 \mathrm{sec}$ ). The test map was identical to the presentation map but contained no landmark symbols. The experimenter named, in turn, each landmark that was to be reached next in tests as well as in presentation, so that only 
the locations of the landmarks, but not their names or their order, had to be remembered.

For recall, the participants were asked to stop scanning as soon as they thought they had reached the next to-be-remembered location on the route. They were carefully instructed to place the midpoint of the ball of the fingertip precisely on the midpoint of the landmark symbol that had marked that location in presentation.

\section{Specific Procedures for the Four Reference Conditions}

I. Body aligned. Body-centered reference could be used, but there were no external cues. The map was aligned with the participant's body midline in recall tests as in presentations. The locations therefore remained in the same position relative to the body in recall. The surrounding frame was neither mentioned nor scanned at any time.

II. Map rotation. Body-centered reference was disrupted, and there were no external cues. To disturb body-centered reference, the orientation of the map relative to the body was changed for recall by rotating it in a clockwise direction by $90^{\circ}$ in tabletop space. The locations were, therefore, not in the same relation to any body-centered cues in the recall phase as in the presentation phase. The participants were informed beforehand and in the demonstrations that the route in recall would be in a different orientation. They were asked to scan the route in the new orientation and to stop at the remembered locations on that route. Experimenter-guided scanning along the initial path showed the new direction in which the route was to be scanned, to indicate the position of the locations on the route. The condition provided no external cues. The surrounding frame was neither mentioned nor scanned at any time.

III. Map rotation + E-frame. Body-centered reference was disrupted, but external reference was provided. The map was rotated for recall as in Condition 2, and the same procedures were used. But the participants were also instructed to use their other (left) hand concomitantly to scan the raised-line frame surrounding the map, in order to relate the target locations to that frame (Figure 1). Using the right hand for the display and the left hand to scan the frame had previously been found the most efficient combination for righthanded people (Millar \& Al-Attar, 2000). Scanning the frame relative to the route and to landmark locations was demonstrated in the two demonstration trials. The frame was used during presentation and in tests. The instructions stressed that landmark locations should be remembered in relation to the frame.

IV. Body aligned + E-frame. Both body-centered reference and external reference cues could be used. The map-to-body orientation remained the same in recall as for Condition 1 . In addition, the participants received the same instructions for use of the frame relative to the route and landmark locations as the participants in Condition 3.

\section{Scoring}

The grid lines on the test map and route and numeral and ruler marks (in centimeters) at the four sides showed the point at which the participant placed the midpoint of the fingertip to indicate the remembered location. The experimenter recorded that point on an exact replica of the map for every location on every trial. The grid record (centimeters and millimeters) showed the precise grid distance of the remembered location from the correct locations on the route. The correct location used was the midpoint of the landmark at that location that the participant had been asked to remember. The distance between the midpoint of the fingertip placed at the remembered location and the correct location was the error measure (in centimeters, regardless of sign) for every location in every trial.

\section{Results}

The comparisons between the reference conditions that tested the predictions from the two hypotheses supported the reference hypothesis. The findings for each prediction will be detailed after an overall analysis, conducted at the suggestion of the editor.

An overall reference conditions $(4) \times$ locations $(5)$ ANOVA (means and standard deviationsin Table 1) showed a significant effect of the reference conditions $[F(3,36)=$ $31.81, p<.0001]$. Locations also differed significantly $[F(4,144)=28.47, p<.0001]$ and interacted significantly with reference condition $[F(12,144)=2.34, p<.009]$.

The interaction was analyzed further by comparisons of the reference conditions that were needed to test the predictions. The comparisons (see below) showed the location effect but no significant interaction with reference conditions, except in the map rotation condition, as compared with the body-aligned condition.

\section{Body Aligned Versus Map Rotation}

The results of the comparison supported the prediction that disturbing body-centered coding, by changing the relation of the route to the body in recall, would increase errors significantly at all locations, consistent with the assumption that accuracy relates to body-centered coding also for a sequence of locations.

The ANOVA showed that errors (Table 1) under map rotation were significantly higher than those in the bodyaligned conditions $[F(1,18)=31.11, p<.0001]$. Locations differed significantly $[F(4,72)=17.23, p<.0001]$ and interacted with the reference conditions $[F(4,72)=$ $2.92, p<.027]$.

All location errors were significantly higher in the map rotation condition than in the body-aligned condition, as was predicted. The location effect was due to relatively higher errors for middle than for end locations (Newman Keuls, $p<.01$ for Locations 2, 3, and 4 vs. Locations 5 and 1, under body alignment, and vs. Location 1 also under map rotation). The interaction was due mainly to increased errors for Location 1 under map rotation [lower than Locations 3 and $4(p<.05)$ but equal to Location 2 and higher than Location $5(p<.05)]$.

Prima facie, the patterns of location errors resemble curves found in short-term verbal memory. But they could not be explained by either naming or serial order effects, since the experimenter named each location in turn first (see the Method section). But the error patterns were consistent with the use of fortuitous but distinctive local touch cues from the route in this map. The middle locations were farthest from salient local touch cues that could have provided additional anchor cues for the recall movements. But the final landmark was just beyond the end of the raised-line route. The change, in touch, to the smooth blank beyond could, in principle, constitute an additional retrieval cue, thus producing more accurate, although not error-free, recall. The first location was a short distance from a distinctive sharp (acute) turn in the raised-line route. The distance from the location would make it a less precise but, nevertheless, usable additional retrieval cue for the first location. An angular turn would be less useful under map rotation. The interpretation is also consis- 
tent with the result for Location 1 in that condition, shown by the analysis of the interaction.

The interpretation of the location effect will be briefly considered later, together with the results from the other comparisons.

\section{Body Aligned Versus Body Aligned + E-Frame}

The reference hypothesis predicts that spatial coding is more accurate when external reference information is added to intact body-centered information than for intact body-centered reference alone. The comparison of the body-aligned and the body-aligned + E-frame conditions supported the prediction.

The ANOVA on errors (Table 1) showed that the participants in the body-aligned + E-frame condition were significantly more accurate than those in the body-aligned condition alone $[F(1,18)=27.23, p<.0001]$, as was predicted. The location effect was significant $[F(4,72)=$ $16.82, p<.0001]$ but did not interact significantly with the reference conditions. Errors were higher for middle locations than for Locations 5 and $1(p<.01$ and $p<.05$, respectively, on post hoc test; also for the body-aligned + E-frame reference condition).

There was no support for the alternative hypothesis that adding external reference would either be deleterious or have no effect. The overall mean error in the body-aligned + E-frame condition was almost half that in the bodyaligned (no-frame) condition (see below).

\section{Map Rotation Versus Map Rotation + E-Frame}

The reference hypothesis implies that external reference information alone can be used to code haptic locations spatially when that information is made available. External frame information was dissociated from bodycentered reference by using map rotation, which disrupts body-centered reference, concomitantly with instructions to use cues from the external frame for reference. The results confirmed the prediction that E-frame information significantly reduces errors when body-centered reference is disturbed, as compared with map rotation conditions that disrupt body-centered reference and provide no external reference information.

The map rotation/map rotation + E-frame $\times$ locations ANOVA on errors (Table 1 ) showed that the reference effect was significant $[F(1,18)=22.34, p<.0001]$, as was predicted. Locations differed significantly $[F(4,72)=$ $22.34, p<.000]$. There was no significant interaction. The location effect for map rotation [higher for Locations 1,2 , and 3 than for Location $5(p<.01)$ and higher than for Location $1(p<.05)$ ] showed the same error patterns.

The reference effect shown here provides decisive evidence that the participants did indeed use the external frame for reference for the locations. The overall mean error was almost twice as large in the map rotation (noframe) condition than in the map rotation + E-frame condition. The result could not have been obtained if the reduction in errors was simply due to the participants' using both hands and so obtaining twice as many egocentric cues for reference (as was suggested by one referee). If so, map rotation should have produced twice the error rate, since it changes the relation of any body-centered cue to the to-be-remembered locations. In fact, the frame condition halved the error rate under map rotation.

\section{Map Rotation + E-Frame Versus \\ Body Aligned + E-Frame}

The comparison tested the final prediction implied by the reference hypothesis-namely, that combined external and body-centered references would produce significantly more accurate spatial coding than would external reference alone. The result supported that prediction also.

Locations differed significantly $[F(4,72)=15.10, p<$ $.0001]$ but produced no interaction in the ANOVA on errors (Table 1). The reference effect was significant $[F(1,18)=$ $18.40, p<.0001]$, as was predicted. The participants in the body-aligned + E-frame condition were nearly twice as accurate as those in the map rotation + E-frame condition.

Additive effects. The results of all the analyses were consistent with the effects of body-centered and external reference cues predicted by the reference hypothesis. But the highly significant differences between reference conditions, shown by the comparison of separate and combined reference conditions at all locations, also suggest strongly that the two forms of coding had additive effects on spatial processing. The additive relation between the conditions is shown clearly by the mean errors, averaged over locations, for all conditions, as is shown in Figure 2.

The graph shows double the errors for the condition that lacked both external and body-aligned reference (II) than for the body-aligned condition without external cues (I)

Table 1

Mean Errors (in Centimeters) and Standard Deviations for Five Locations: 1 (Bank), 2 (Supermarket), 3 (Railway Station), 4 (Friend's House), and

5 (Home), in the Body-Aligned, Map Rotation, Map Rotation + E-Frame, and Body-Aligned + E-Frame Reference Conditions

\begin{tabular}{|c|c|c|c|c|c|c|c|c|c|c|}
\hline \multirow{3}{*}{$\begin{array}{l}\text { Reference } \\
\text { Conditions }\end{array}$} & \multicolumn{10}{|c|}{ Landmark Locations } \\
\hline & \multicolumn{2}{|c|}{1} & \multicolumn{2}{|c|}{2} & \multicolumn{2}{|c|}{3} & \multicolumn{2}{|c|}{4} & \multicolumn{2}{|c|}{5} \\
\hline & $M$ & $S D$ & $M$ & $S D$ & $M$ & $S D$ & $M$ & $S D$ & $M$ & $S D$ \\
\hline Body aligned & 0.25 & 0.14 & 0.47 & 0.13 & 0.43 & 0.21 & 0.69 & 0.43 & 0.14 & 0.09 \\
\hline Map rotation & 0.72 & 0.32 & 0.69 & 0.24 & 1.12 & 0.46 & 1.01 & 0.31 & 0.47 & 0.22 \\
\hline Map rotation + E-fram & 0.37 & 0.19 & 0.55 & 0.31 & 0.62 & 0.36 & 0.48 & 0.24 & 0.10 & 0.03 \\
\hline Body aligned + E-frame & 0.15 & 0.10 & 0.24 & 0.15 & 0.34 & 0.21 & 0.32 & 0.15 & 0.06 & 0.05 \\
\hline Overall means & \multicolumn{2}{|c|}{0.37} & \multicolumn{2}{|c|}{0.49} & \multicolumn{2}{|c|}{0.63} & \multicolumn{2}{|c|}{0.63} & \multicolumn{2}{|c|}{0.19} \\
\hline
\end{tabular}




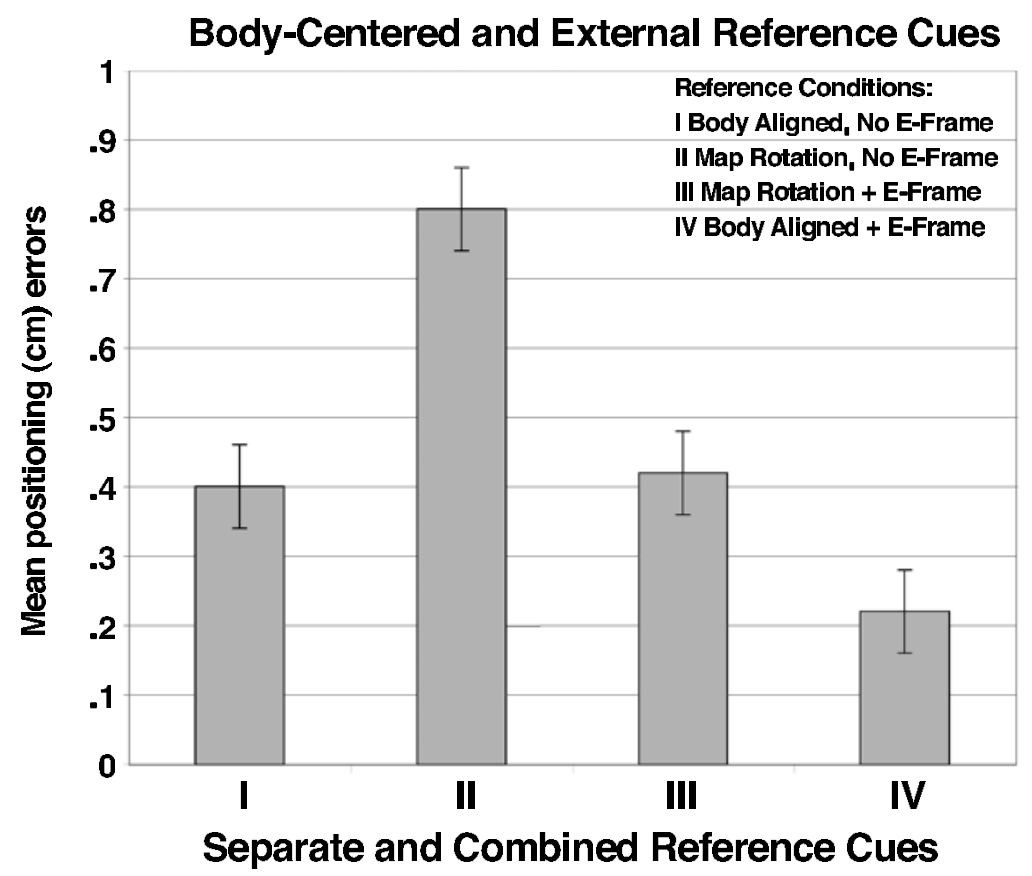

Figure 2. Mean errors and standard errors of the means, suggesting additive relations between the reference conditions.

or for the external reference condition when body-centered alignment was disturbed (III). The conjunction of external reference and intact body-centered alignment (IV) produced only half the errors found with either form of reference alone (II or III).

Location effects. The interpretation (see above) that the location effect was due to fortuitous but salient local touch cues from the route in the map used here is consistent with the fact that the effect was due to the same basic error pattern in all reference conditions. The participants' spontaneous (tabulated) comments in the final debriefing session also supported the interpretation. The participants frequently mentioned attempting to use local route features, including turns. The interpretationimplies, of course, that error patterns for an irregularly placed sequence of locations depends on the specific layout being used and on the salient features near target locations that the layout happens to provide.

What the significant location effect here does suggest is that people also use fortuitous local touch cues even when they clearly also use body-centered and external frame cues for reference at every location.

\section{SUMMARY AND GENERAL DISCUSSION}

The aim of the study was to investigate how bodycentered reference and external reference information affect spatial coding of an irregular sequence of locations scanned by touch and movement without inputs from distal modalities. Separating and combining the two forms of reference produced important new findings. The comparisons suggested that the two forms of reference have independent additive effects on spatial coding. Combining external frame information with intact body-centered reference made spatial coding of locations twice as accurate as conditions either with intact body-centered reference alone or with external reference alone when body-centered reference was disturbed.

The irregular sequence of locations, used here to test additionally how far spatial coding of more than one location would occur also for purely haptic inputs (see the introduction), did indeed show significantly greater accuracy at all locations with added external, as well as with intact body-centered, information. The results also showed a significant locations effect. The internal evidence suggested that this was due to fortuitous local touch features on the route in the map used here. This interpretation implies that error patterns for different layouts are likely to differ with the number, position, and salience of local touch cues in the different spatial layouts.

The location effect found here is nevertheless important, because it suggests that people use fortuitous local touch cues from the layouts, as well as body-centered and external frame cues, for irregular patterns of haptic locations. How specific fortuitous local cues affect error patterns and how they relate to the scanning movements that are crucial in haptic perception has yet to be investigated for irregular patterns of haptic locations.

\section{Additive Effects}

The finding that external cues beyond target locations can be as effective as body-centered reference cues in haptic conditions has practical implications. Efficient exploration of small-scale tactile layouts and maps requires ex- 
perience or assisted learning in blind conditions (Foulke, 1982; Millar, 1997; Ungar et al., 1997). Surrounding frames may need to be produced and signaled explicitly for inexperienced people. This study provides new empirical evidence that concomitantly using an external frame significantly improves recall of target locations, even when the display is displaced relative to the body. The advantage shown here of combining intact body-centered information with external frame cues is likely to be particularly important for translating locations encountered in smallscale displays into landmarks that can be used for orientation in large-scale environments.

Theoretically, the additive effects suggested by the separate and combined error results imply that the two forms of spatial organization are largely independent. Neurophysiological studies also suggest a dissociation. Egocentric and allocentric spatial performance is associated with different brain regions and neuronal circuits (see, e.g., Arbib, 1991; Bayliss \& Moore, 1994; Berthoz, 1991; Rolls, 1991; Stein, 1992; Vallar, Lobel, Galati, Berthoz, Pizzamilio, \& Le Bihan, 1999). Snyder and colleagues (Snyder, Grieve, Brotchie, \& Andersen, 1998) found almost completely segregated populations of cells for worldreferenced and body-referenced (retinal) visual signals in the parietal cortex of rhesus monkeys. Their findings were for vision. But the cortical and subcortical areas involved in diverse spatial task performance receive inputs from most sensory sources, including touch (see, e.g., Andersen, Snyder, Bradley, \& Xing, 1997; Arbib, 1991; Berthoz, 1991; Paillard, 1991; Rains \& Milner, 1994; Ratcliff, 1991; Stein, 1992). The suggestion (Snyder et al., 1998) that segregated streams of information have the biological advantage that their combination increases processing efficiency would explain the considerably greater efficiency that the combined reference conditions produced here.

\section{Implications for Spatial Coding}

The results were consistent with the effects predicted by the reference hypothesis. They were not compatible with the view that haptic inputs can be coded spatially only by body-centered reference and that the use of external reference cues requires vision or a distal modality. When external reference beyond the locations was made available concomitantly, it had similar effects on recall as the presence of intact body-centered cues. Explicit external reference cues decreased errors even though map rotation disturbed whatever body-centered cues were used for reference in presentation.

Modalities differ in the extent to which they provide cues from different sources routinely. Cues that are external to a new haptic location have to be sought, or explicitly provided, whether they are potentially within reach or beyond immediate reach. Moreover, haptic inputs depend crucially on scanning movements. The point is that when explicit reference information was provided, it was used. The function of external cues in reducing errors can be described as follows. The location of the landmark felt by the scanning finger is related to the frame, as indicated by the other hand. In recall, the frame provides retrieval cues that stop the positioning movement more accurately at the correct location, even when egocentric reference is disturbed.

The findings are consistent with the view that the data are described better by assuming active processing of whatever inputs can be used as reference cues for targets (Millar, 1994, 1997, 2000) than by the assumption of modalityspecific spatial stores. It is also relevant to the difference between using body-centered (retinal or viewer-centered) cues and using an external surround for reference (Hinton \& Parsons, 1988; Snyder et al., 1998) in vision. This view suggests that the processing activities, which organize inputs for reference, are similar or in common in vision and touch, and that the form of spatial reference is a function of the information that is available from diverse sources (Millar \& Al-Attar, 2002) in different task conditions.

This new evidence shows that external, as well as bodycentered, reference affects spatial coding of haptic locations and suggests that they have additive effects.

\section{REFERENCES}

AdAMs, J. A., \& DiJKstra, S. (1966). Short-term memory for motor responses. Journal of Experimental Psychology, 71, 314-318.

Andersen, R. A., Snyder, L. H., Bradley, D. C., \& Xing, J. (1997). Multimodal representation of space in the posterior parietal cortex and its use in planning movements. Annual Review of Neuroscience, 20, 303-330.

ARBIB, M. A. (1991). Interaction of multiple representations of space in the brain. In J. Paillard (Ed.), Brain and space (pp. 379-403). Oxford: Oxford University Press.

BAdDEley, A. D. (1990). Human memory: Theory and practice. Hillsdale, NJ: Erlbaum.

BAdDEley, A. D. (2000). The episodic buffer: A new component of working memory? Trends in Cognitive Sciences, 4, 417-422.

Baddeley, A. D., \& Hitch, G. (1974). Working memory. In G. H. Bower (Ed.), The psychology of learning and motivation (Vol. 8, pp. 47-89). New York: Academic Press.

Ballesteros, S., Millar, S., \& Reales, J. M. (1998). Symmetry in haptic and in visual shape perception. Perception \& Psychophysics, 60, 389-404.

Barrett, D. J. K., Bradshaw, M. F., Rose, D., Everatt, P. J., \& SimpSON, P. J. (2001). Reflexive shifts of attention operate in an egocentric coordinate frame. Perception, 30, 1083-1091.

Bayliss, G. C., \& Moore, B. O. (1994). Hippocampal lesions impair spatial response selection in the primate. Experimental Brain Research, 98, 110-118.

BERTHOZ, A. (1991). Reference frames for the perception and control of movements. In J. Paillard (Ed.), Brain and space (pp. 81-111). Oxford: Oxford University Press.

Carpenter, P. A., \& Eisenberg, P. (1978). Mental rotation and the frame of reference in blind and sighted individuals. Perception \& Psychophysics, 23, 117-124.

Foulke, E. (1982). Reading Braille. In W. Schiff \& E. Foulke (Eds.), Tactual perception: A source book (pp. 168-208). Cambridge: Cambridge University Press.

Hasbroucq, T., \& Guiard, Y. (1986). Response determination in tactile motor tasks: Body-vs device-centered cues. Cahiers de Psychologie Cognitive/Current Psychology of Cognition, 6, 367-377.

HATWELL, Y. (1960). Étude de quelques illusions géométriques tactiles chez les aveugles. L'Anneé Psychologique, 60, 11-27.

Heller, M. A., Calcaterra, J. A., Green, S. L., \& Barnette, S. L. (1999). Perception of the horizontal and vertical in tangible displays: Minimal gender differences. Perception, 28, 387-394.

Helstrup, T. (2001). Concurrent and retroactive interference effects in memory for movement patterns. Quarterly Journal of Experimental Psychology, 54A, 547-560.

Hinton, G. E., \& PARsons, L. M. (1988). Scene-based and viewercentered representations for comparing shapes. Cognition, 30, 1-35. 
Hollins, M., \& Kelley, E. K. (1988). Spatial updating in blind and sighted people. Perception \& Psychophysics, 43, 380-388.

JoHNSON, P. (1982). The functional equivalence of imagery and movement. Quarterly Journal of Experimental Psychology, 34A, 349-365.

KAHNEMAN, D., SLOVIC, P., \& TVERSKY, A. (1982). Judgment under uncertainty: Heuristics and biases. New York: Cambridge University Press.

LAABS, G. L. (1973). Retention characteristics of different reproduction cues in short-term memory. Journal of Experimental Psychology, 100, 166-177.

LAabs, G. L., \& Simmons, R. W. (1981). Motor memory. In D. Holding (Ed.), Human skills (pp. 119-151). Chichester, U.K.: Wiley.

LECHE LT, E. C. (1982). The stability of tactile laterality differences across manipulations of temporal and spatial stimulus patterning. International Journal of Rehabilitation Research, 5, 370-372.

Millar, S. (1975). Spatial memory by blind and sighted children. British Journal of Psychology, 66, 449-459.

MiLlar, S. (1976). Spatial representation by blind and sighted children. Journal of Experimental Child Psychology, 21, 460-479.

MiLlaR, S. (1978). Aspects of information from touch and movement. In G. Gordon (Ed.), Active touch: The mechanisms of recognition of objects by manipulation (pp. 215-227). Oxford: Pergamon Press.

MiLlar, S. (1979). The utilisation of shape and movement cues in simple spatial tasks by blind and sighted children. Perception, 8, 11-20.

Millar, S. (1981). Self-referent and movement cues in coding spatial location by blind and sighted children. Perception, 10, 255-264.

MiLlaR, S. (1985). Movement cues and body orientation in recall of location by blind and sighted children. Quarterly Journal of Experimental Psychology, 37A, 257-279.

MiLlaR, S. (1988). Models of sensory deprivation: The nature/nurture dichotomy and spatial representation in the blind. International Journal of Behavioural Development, 11, 69-87.

MiLlaR, S. (1991). A reverse lag in the recognition and production of tactual drawings: Theoretical implications for haptic coding. In M. A. Heller \& W. Schiff (Eds.), The psychology of touch (pp. 301-325). Hillsdale, NJ: Erlbaum.

MILlAR, S. (1994). Understanding and representing space: Theory and evidence from studies with blind and sighted children. Oxford: Oxford University Press, Clarendon Press.

MiLlaR, S. (1997). Reading by touch. London: Routledge.

MILLAR, S. (2000). Modality and mind: Convergent active processing in interrelated networks: A model of development and perception by touch. In M. A. Heller (Ed.), Touch, representation and blindness (pp. 99-141). Oxford: Oxford University Press.

Millar, S., \& Al-ATtAR, Z. (2000). Vertical and bisection bias in active touch. Perception, 29, 481-500.

MiLlar, S., \& AL-ATTAR,Z. (2001). Illusions in reading maps by touch. British Journal of Psychology, 92, 643-657.

MiLlar, S., \& AL-ATTAR,Z. (2002). The Müller-Lyer illusion in touch and vision: Implications for multisensory processes. Perception \& Psychophysics, 64, 353-365.

Millar, S., \& ITTYERAH, M. (1991). Mental practice without visuospatial information. International Journal of Behavioral Development, 15, 125-146.

OldField, S. R., \& Philips, J. R. (1983). The spatial characteristics of tactile form perception. Perception, 12, 615-626.

PAillard, J. (1991). Motor and representational framing of space. In J. Paillard (Ed.), Brain and space (pp. 163-182). Oxford: Oxford University Press.

PAShler, H. (1990). Coordinate frame for symmetry detection and object recognition. Journal of Experimental Psychology: Perception \& Performance, 16, 150-163.

RAINS, G. D., \& MILNER, B. (1994). Right-hippocampal contralateral hand effect in the recall of spatial location in the tactual modality. Neuropsychologia, 32, 1233-1242.

RATCLIFF, G. (1991). Brain and space: Some deductions from clinical evidence. In J. Paillard (Ed.), Brain and space (pp. 237-250). Oxford: Oxford University Press.

Re VESZ, G. (1950). Psychology and art of the blind. London: Longmans. RoLls, E. T. (1991). Functions of the primate hippocampus in spatial processing and memory. In J. Paillard (Ed.), Brain and space (pp. 353376). Oxford: Oxford University Press.

Rossano, M. J., \& WARREN, D. H. (1989a). The importance of alignment in blind subjects' use of tactual maps. Perception, 18, 805-816.

Rossano, M. J., \& WARren, D. H. (1989b). Misaligned maps lead to predictable errors. Perception, 18, 215-229.

SHE PARD, R. N., \& COOPER, L. (1982). Mental images and their transformations. Cambridge, MA: MIT Press.

ShePARd, R. N., \& Metzler, J. (1971). Mental rotation of threedimensional objects. Science, 171, 701-703.

Snyder, L. H., Grieve, K. L., Brotchie, P., \& Andersen, R. A. (1998). Separate body and world-referenced representations of visual space in the parietal cortex. Nature, 394, 887-891.

STEIN, J. F. (1992). The representation of egocentric space in the posterior parietal cortex. Behavioral \& Brain Sciences, 15, 691-700.

Ungar, S., Blades, M., \& Spencer, C. (1997). Strategies for knowledge acquisition from cartographic maps by blind and visually impaired adults. Cartographic Journal, 34, 93-110.

Vallar, G., Lobel, E., Galati, G., Berthoz A., Pizzamilio, L., \& Le BIHAN, D. (1999). A fronto-parietal system for computing the egocentric frame of reference. Experimental Brain Research, 124, 281286.

WARREN, D. H. (1977). Blindness and early childhood development. New York: American Foundation for the Blind.

(Manuscript received June 6, 2002: revision accepted for publication May 25, 2003.) 